\title{
On the Location of the Zeros of Certain Polynomials
}

\section{M.H.Gulzar ${ }^{1}$, Rubia Akhter ${ }^{2}$}

Department of Mathematic, University of Kashmir, Srinagar 190006

\section{Abstract:}

In this paper we generalize Bairagi, Jain, Mishra and Saha's result that the zeros of a polynomial $P(z)=\sum_{j=0}^{n} a_{j} z^{j}$ with $t\left|a_{j}\right| \geq\left|a_{j-1}\right|, j=2,3, \ldots ., n$ for some $\mathrm{t}>0$ and $\left|A \operatorname{Arg} a_{j}-\beta\right| \leq \alpha \leq \frac{\pi}{2}, j=1,2, \ldots . ., n$ for some real $\alpha, \beta$, of modulus greater than

$$
\frac{t(n-1)}{n}\left\{\cos \alpha+\sin \alpha+\frac{2 \sin \alpha}{n\left|a_{n}\right|} \sum\left(\frac{n}{t(n-1)}\right)^{n-1-j}(j+1)\left|a_{j+1}\right|\right\}
$$

are simple, and other similar results to polynomials with lesser restrictive conditions on their coefficients.

\section{Mathematics Subject Classification: 30C10, 30C15.}

Key Words and Phrases: Bound, Coefficient, Polynomial, Zeros.

\section{Introduction}

About the order of certain zeros of a polynomial, Aziz and Mohammad [1] proved the following result.

Theorem A. Let $P(z)=\sum_{j=0}^{n} a_{j} z^{j}$ be a polynomial of degree $\mathrm{n}$ such that

$$
a_{n} \geq a_{n-1} \geq \ldots \ldots \geq a_{1} \geq a_{0}>0 \text {. }
$$

Then all the zeros of $\mathrm{P}(\mathrm{z})$ of modulus greater than or equal to $\frac{n}{n+1}$ are simple.

In [2], Aziz and Mohammad gave a generalization as well a refinement of Theorem A as follows.

Theorem B. Let $P(z)=\sum_{j=0}^{n} a_{j} z^{j}$ be a polynomial of degree $\mathrm{n}>1$ such that for some $\mathrm{t}>0$, $t a_{j} \geq a_{j-1}>0, j=2,3, \ldots . ., n, a_{0}$ may be a real or complex number.Then all the zeros of $\mathrm{P}(\mathrm{z})$ of modulus greater than $\frac{t(n-1)}{n}$ are simple.

Bairagi, Jain, Mishra and Saha [3] extended Theorem B to polynomials with complex coefficients and proved the following result.

Theorem C. Let $P(z)=\sum_{j=0}^{n} a_{j} z^{j}$ be a polynomial of degree $\mathrm{n}>1$ such that for some $\mathrm{t}>0$,

$t\left|a_{j}\right| \geq\left|a_{j-1}\right|, j=1,2, \ldots . ., n$ and for some real $\alpha, \beta,\left|\operatorname{Arg} a_{j}-\beta\right| \leq \alpha \leq \frac{\pi}{2}, j=1,2, \ldots . ., n$.

Then $\mathrm{P}(\mathrm{z})$ cannot have a zero of order $\geq 2$ with modulus greater than 


$$
\frac{t(n-1)}{n}\left\{\cos \alpha+\sin \alpha+\frac{2 \sin \alpha}{n\left|a_{n}\right|} \sum_{j=0}^{n-2}\left(\frac{n}{t(n-1)}\right)^{n-1-j}(j+1)\left|a_{j+1}\right|\right\} .
$$

In other words, all the zeros of $\mathrm{P}(\mathrm{z})$ with modulus greater than

$$
\frac{t(n-1)}{n}\left\{\cos \alpha+\sin \alpha+\frac{2 \sin \alpha}{n\left|a_{n}\right|} \sum_{j=0}^{n-2}\left(\frac{n}{t(n-1)}\right)^{n-1-j}(j+1)\left|a_{j+1}\right|\right\}
$$

are simple.

In the same paper they also proved the following result.

Theorem D. Let $P(z)=\sum_{j=0}^{n} a_{j} z^{j}$ be a polynomial of degree $\mathrm{n}>1$ with $\operatorname{Re} a_{j}=\alpha_{j}, \operatorname{Im} a_{j}=\beta_{j}$, $\mathrm{j}=0,1, \ldots, \mathrm{n}$. If for some $\mathrm{t}>0$

$$
t \alpha_{j} \geq \alpha_{j-1} \geq 0, j=2,3, \ldots . ., n \text { and } \alpha_{n}>0,
$$

then $\mathrm{P}(\mathrm{z})$ cannot have a zero of order $\geq 2$ with modulus greater than

$$
\frac{t(n-1)}{n}\left\{1+\frac{2}{n \alpha_{n}} \sum_{j=0}^{n-1}\left(\frac{n}{t(n-1)}\right)^{n-j}(j+1)\left|\beta_{j+1}\right|\right\} .
$$

In other words, all the zeros of $\mathrm{P}(\mathrm{z})$ with modulus greater than

$$
\frac{t(n-1)}{n}\left\{1+\frac{2}{n \alpha_{n}} \sum_{j=0}^{n-1}\left(\frac{n}{t(n-1)}\right)^{n-j}(j+1)\left|\beta_{j+1}\right|\right\}
$$

are simple.

\section{Main Resuls}

In this paper we prove the following generalizations of Theorems $\mathrm{C}$ and $\mathrm{D}$.

Theorem 1. Let $P(z)=\sum_{j=0}^{n} a_{j} z^{j}$ be a polynomial of degree $\mathrm{n}>1$ such that for some $\mathrm{t}>0, k \geq 1$,

$$
k\left|a_{n}\right| \geq \frac{1}{t}\left|a_{n-1}\right| \geq \frac{1}{t^{2}}\left|a_{n-2}\right| \geq \ldots \ldots . . \geq \frac{1}{t^{n-1}}\left|a_{1}\right| \geq \frac{1}{t^{n}}\left|a_{0}\right|
$$

and for some real $\alpha, \beta,\left|\operatorname{Arg} a_{j}-\beta\right| \leq \alpha \leq \frac{\pi}{2}, j=1,2, \ldots . ., n$.

Then all the zeros of $\mathrm{P}(\mathrm{z})$ with modulus greater than

$$
\frac{t(n-1)}{n\left|a_{n}\right|}\left\{k\left|a_{n}\right|(\cos \alpha+\sin \alpha+1)-\left|a_{n}\right|+\frac{2 \sin \alpha}{n} \sum_{j=0}^{n-2}\left(\frac{n}{t(n-1)}\right)^{n-1-j}(j+1)\left|a_{j+1}\right|\right\}
$$

are simple.

Remark 1. For $\mathrm{k}=1$, Theorem 1 reduces to Theorem C.

Theorem 2. Let $P(z)=\sum_{j=0}^{n} a_{j} z^{j}$ be a polynomial of degree $\mathrm{n}>1$ with $\operatorname{Re} a_{j}=\alpha_{j}, \operatorname{Im} a_{j}=\beta_{j}$, $\mathrm{j}=0,1, \ldots, \mathrm{n}$. If for some $\mathrm{t}>0$ and $k \geq 1$,

$$
k \alpha_{n} \geq \frac{1}{t} \alpha_{n-1} \geq \frac{1}{t^{2}} \alpha_{n-2} \geq \ldots \ldots \geq \frac{1}{t^{n-1}} \alpha_{1} \geq \frac{1}{t^{n}} \alpha_{0} \geq 0, \quad \text { and } \alpha_{n}>0,
$$

then $\mathrm{P}(\mathrm{z})$ cannot have a zero of order $\geq 2$ with modulus greater than 


$$
\frac{t(n-1)}{n}\left\{2 k-1+\frac{2}{n \alpha_{n}} \sum_{j=0}^{n-1}\left(\frac{n}{t(n-1)}\right)^{n-j}(j+1)\left|\beta_{j+1}\right|\right\} .
$$

In other words, all the zeros of $\mathrm{P}(\mathrm{z})$ with modulus greater than

$$
\frac{t(n-1)}{n}\left\{2 k-1+\frac{2}{n \alpha_{n}} \sum_{j=0}^{n-1}\left(\frac{n}{t(n-1)}\right)^{n-j}(j+1)\left|\beta_{j+1}\right|\right\}
$$

are simple.

Remark 2. For $\mathrm{k}=1$, Theorem 2 reduces to Theorem D.

Applying Theorem 2 to the polynomial $-\mathrm{iP}(\mathrm{z})$, we get the following result.

Corollary 1. Let $P(z)=\sum_{j=0}^{n} a_{j} z^{j}$ be a polynomial of degree $\mathrm{n}>1$ with $\operatorname{Re} a_{j}=\alpha_{j}, \operatorname{Im} a_{j}=\beta_{j}$, $\mathrm{j}=0,1, \ldots, \mathrm{n}$. If for some $\mathrm{t}>0$ and $k \geq 1$,

$$
k \beta_{n} \geq \frac{1}{t} \beta_{n-1} \geq \frac{1}{t^{2}} \beta_{n-2} \geq \ldots \ldots \geq \frac{1}{t^{n-1}} \beta_{1} \geq \frac{1}{t^{n}} \beta_{0} \geq 0, \quad \text { and } \beta_{n}>0,
$$

then $\mathrm{P}(\mathrm{z})$ cannot have a zero of order $\geq 2$ with modulus greater than

$$
\frac{t(n-1)}{n}\left\{2 k-1+\frac{2}{n \beta_{n}} \sum_{j=0}^{n-1}\left(\frac{n}{t(n-1)}\right)^{n-j}(j+1)\left|\alpha_{j+1}\right|\right\} .
$$

In other words, all the zeros of $\mathrm{P}(\mathrm{z})$ with modulus greater than

$$
\frac{t(n-1)}{n}\left\{2 k-1+\frac{2}{n \beta_{n}} \sum_{j=0}^{n-1}\left(\frac{n}{t(n-1)}\right)^{n-j}(j+1)\left|\alpha_{j+1}\right|\right\}
$$

are simple.

\section{Lemmas}

For the proofs of the above results we need the following lemmas.

Lemma 1. For any two complex numbers $z_{1}, z_{2}$ with $\left|z_{1}\right| \geq\left|z_{2}\right|$ and $\left|\operatorname{Arg} z_{j}-\beta\right| \leq \alpha \leq \frac{\pi}{2}, j=1,2$, for some real $\alpha, \beta$,

$$
\left|z_{1}-z_{2}\right| \leq\left(\left|z_{1}\right|-\left|z_{2}\right|\right) \cos \alpha+\left(\left|z_{1}\right|+\left|z_{2}\right|\right) \sin \alpha \text {. }
$$

Lemma 1 is due to Govil and Rahman [4].

Lemma 2. Let $P(z)=\sum_{j=0}^{n} a_{j} z^{j}$ be a polynomial of degree $\mathrm{n}$ such that for some real $\alpha, \beta$,

$$
\left|A r g a_{j}-\beta\right| \leq \alpha \leq \frac{\pi}{2}, j=1,2, \ldots \ldots, n,
$$

and

$$
k\left|a_{n}\right| \geq\left|a_{n-1}\right| \geq \ldots \ldots . \geq\left|a_{1}\right| \geq\left|a_{0}\right| .
$$

Then $\mathrm{P}(\mathrm{z})$ has all its zeros in

$$
|z| \leq \frac{1}{\left|a_{n}\right|}\left\{k\left|a_{n}\right|(\cos \alpha+\sin \alpha+1)-\left|a_{n}\right|+2 \sin \alpha \sum_{j=0}^{n-1}\left|a_{j}\right|\right\} .
$$

Proof of Lemma2. Consider the polynomial

$$
F(z)=(1-z) P(z)
$$




$$
\begin{aligned}
& =-a_{n} z^{n+1}+\left(a_{n}-a_{n-1}\right) z^{n}+\ldots \ldots+\left(a_{1}-a_{0}\right) z+a_{0} \\
& =-a_{n} z^{n+1}-(k-1) a_{n} z^{n}+\left(k a_{n}-a_{n-1}\right) z^{n}+\ldots \ldots+\left(a_{1}-a_{0}\right) z+a_{0}
\end{aligned}
$$

For $|z|>1$, we have by using the hypothesis and Lemma 1,

$$
\begin{aligned}
|F(z)| & \geq\left|a_{n} \| z\right|^{n}\left[|z+k-1|-\frac{1}{\left|a_{n}\right|}\left\{\left|k a_{n}-a_{n-1}\right|+\frac{\left|a_{n-1}-a_{n-2}\right|}{|z|}+\ldots+\frac{\left|a_{1}-a_{0}\right|}{|z|^{n-1}}+\frac{\left|a_{0}\right|}{|z|^{n}}\right\}\right] \\
> & \left.\left|a_{n}\right||z|^{n}\left[|z+k-1|-\frac{1}{\left|a_{n}\right|}\left\{\left|k a_{n}-a_{n-1}\right|+\left|a_{n-1}-a_{n-2}\right|+\ldots .+\left|a_{1}-a_{0}\right|+\left|a_{0}\right|\right\}\right]\right] \\
& \geq\left|a_{n}\right| \|\left. z\right|^{n}\left[|z+k-1|-\frac{1}{\left|a_{n}\right|}\left\{\left(k\left|a_{n}\right|-\left|a_{n-1}\right|\right) \cos \alpha+\left(k\left|a_{n}\right|+\left|a_{n-1}\right|\right) \sin \alpha\right.\right. \\
& =\left|a_{n} \| z\right|^{n}\left[|z+k-1|-\frac{1}{\left|a_{n}\right|}\left\{k\left|a_{n}\right|(\cos \alpha+\sin \alpha)-\left|a_{0}\right|(\cos \alpha+\sin \alpha-1)+2 \sin \alpha \sum_{j=0}^{n-1}\left|a_{j}\right|\right\}\right] \\
& \geq\left|a_{n} \| z\right|^{n}\left[|z+k-1|-\frac{1}{\left|a_{n}\right|}\left\{k\left|a_{n}\right|(\cos \alpha+\sin \alpha)+2 \sin \alpha \sum_{j=0}^{n-1}\left|a_{j}\right|\right\}\right] \\
> & 0 \quad+\left(\left|a_{1}\right|-\left|a_{0}\right|\right) \cos \alpha+\left(\left|a_{n-1}\right|+\left|a_{n-2}\right|\right) \sin \alpha+\ldots \ldots
\end{aligned}
$$

if

$$
|z+k-1|>\frac{1}{\left|a_{n}\right|}\left\{k\left|a_{n}\right|(\cos \alpha+\sin \alpha)+2 \sin \alpha \sum_{j=0}^{n-1}\left|a_{j}\right|\right\} .
$$

This shows that the zeros of $\mathrm{F}(\mathrm{z})$ with modulus greater than 1 lie in

$$
|z+k-1| \leq \frac{1}{\left|a_{n}\right|}\left\{k\left|a_{n}\right|(\cos \alpha+\sin \alpha)+2 \sin \alpha \sum_{j=0}^{n-1}\left|a_{j}\right|\right\} \text {. }
$$

Since the zeros of $\mathrm{F}(\mathrm{z})$ with modulus less than or equal to 1 already satisfy the above inequality, it follows that all the zeros of $\mathrm{F}(\mathrm{z})$ and hence $\mathrm{P}(\mathrm{z})$ lie in

$$
|z+k-1| \leq \frac{1}{\left|a_{n}\right|}\left\{k\left|a_{n}\right|(\cos \alpha+\sin \alpha)+2 \sin \alpha \sum_{j=0}^{n-1}\left|a_{j}\right|\right\} \text {. }
$$

Thus for any zero $\mathrm{z}$ of $\mathrm{P}(\mathrm{z})$, we have

$$
\begin{aligned}
|z| & =|z+k-1-(k-1)| \\
& \leq|z+k-1|+|k-1| \\
& \leq \frac{1}{\left|a_{n}\right|}\left\{k\left|a_{n}\right|(\cos \alpha+\sin \alpha)+2 \sin \alpha \sum_{j=0}^{n-1}\left|a_{j}\right|\right\}+k-1 \\
& =\frac{1}{\left|a_{n}\right|}\left\{k\left|a_{n}\right|(\cos \alpha+\sin \alpha+1)-\left|a_{n}\right|+2 \sin \alpha \sum_{j=0}^{n-1}\left|a_{j}\right|\right\}
\end{aligned}
$$

In other words, all the zeros of $\mathrm{P}(\mathrm{z})$ lie in 


$$
|z| \leq \frac{1}{\left|a_{n}\right|}\left\{k\left|a_{n}\right|(\cos \alpha+\sin \alpha+1)-\left|a_{n}\right|+2 \sin \alpha \sum_{j=0}^{n-1}\left|a_{j}\right|\right\} .
$$

Lemma 3. Let $P(z)=\sum_{j=0}^{n} a_{j} z^{j}$ be a polynomial of degree $\mathrm{n}$ such that for some real $\alpha, \beta$,

$$
\left|\operatorname{Arga}_{j}-\beta\right| \leq \alpha \leq \frac{\pi}{2}, j=1,2, \ldots ., n,
$$

and for some $\mathrm{a}>0$,

$$
k\left|a_{n}\right| \geq a\left|a_{n-1}\right| \geq a^{2}\left|a_{n-2}\right| \geq \ldots . . . \geq a^{n-1}\left|a_{1}\right| \geq a^{n}\left|a_{0}\right| .
$$

Then $\mathrm{P}(\mathrm{z})$ has all its zeros in

$$
|z| \leq \frac{1}{a\left|a_{n}\right|}\left\{k\left|a_{n}\right|(\cos \alpha+\sin \alpha+1)-\left|a_{n}\right|+2 \sin \alpha \sum_{j=0}^{n-1} a^{n-j}\left|a_{j}\right|\right\} .
$$

Proof of Lemma 3. Using the conditions of Lemma 2, we can say that the polynomial P(z/a) satisfies the conditions of Lemma 1 and therefore has all its zeros in

$$
|z| \leq \frac{1}{\left|a_{n}\right|}\left\{k\left|a_{n}\right|(\cos \alpha+\sin \alpha+1)-\left|a_{n}\right|+2 \sin \alpha \sum_{j=0}^{n-1} a^{n-j}\left|a_{j}\right|\right\} .
$$

Accordingly the polynomial $\mathrm{P}(\mathrm{z})=\mathrm{P}(\mathrm{az} / \mathrm{a})$ has all its zeros in

Lemma 4. Let $P(z)=\sum_{j=0}^{n} a_{j} z^{j}$ be a polynomial with $\operatorname{Re} a_{j}=\alpha_{j}, \operatorname{Im} a_{j}=\beta_{j}, j=0,1, \ldots ., n$ such that for some $k \geq 1$,

$$
k \alpha_{n} \geq \alpha_{n-1} \geq \alpha_{n-2} \geq \ldots \ldots . \alpha_{1} \geq \alpha_{0} \geq 0, \text { and } \alpha_{n}>0 .
$$

Then $\mathrm{P}(\mathrm{z})$ has all its zeros in

$$
|z+k-1| \leq k+\frac{2}{\alpha_{n}} \sum_{j=0}^{n}\left|\beta_{j}\right|
$$

Lemma 4 is a simple deduction from a result of Govil and Mc-tume [5] .

Remark 2. For any zero $\mathrm{z}$ of $\mathrm{P}(\mathrm{z})$ of Lemma 4, we have

$$
\begin{aligned}
|z|=|z+k-1-(k-1)| & \leq|z+k-1|+k-1 \\
& \leq k+\frac{2}{\alpha_{n}} \sum_{j=0}^{n}\left|\beta_{j}\right|+k-1 \\
& =2 k-1+\frac{2}{\alpha_{n}} \sum_{j=0}^{n}\left|\beta_{j}\right| .
\end{aligned}
$$

Hence all the zeros of $\mathrm{P}(\mathrm{z})$ of Lemma 3 lie in

$$
|z| \leq 2 k-1+\frac{2}{\alpha_{n}} \sum_{j=0}^{n}\left|\beta_{j}\right| .
$$

Lemma 5. Let $P(z)=\sum_{j=0}^{n} a_{j} z^{j}$ be a polynomial of degree $\mathrm{n}>1$ with $\operatorname{Re} a_{j}=\alpha_{j}, \operatorname{Im} a_{j}=\beta_{j}$, $\mathrm{j}=0,1, \ldots, \mathrm{n}$. If for some $\mathrm{a}>0$ and $k \geq 1$,

$$
k \alpha_{n} \geq a \alpha_{n-1} \geq a^{2} \alpha_{n-2} \geq \ldots . . \geq a^{n-1} \alpha_{1} \geq a^{n} \alpha_{0} \geq 0, \alpha_{n}>0
$$


Then $\mathrm{P}(\mathrm{z})$ has all its zeros in

$$
|z| \leq \frac{1}{a}\left\{2 k-1+\frac{2}{\alpha_{n}} \sum_{j=0}^{n} a^{n-j}\left|\beta_{j}\right|\right\} .
$$

Proof of Lemma 5. Using the conditions of Lemma 4, we can say that the polynomial P(z/a) satisfies the conditions of Lemma 3and therefore has all its zeros in

$$
|z| \leq 2 k-1+\frac{2}{\alpha_{n}} \sum_{j=0}^{n} a^{n-j}\left|\beta_{j}\right| .
$$

Accordingly the polynomial $\mathrm{P}(\mathrm{z})=\mathrm{P}(\mathrm{az} / \mathrm{a})$ has all its zeros in

$$
|z| \leq \frac{1}{a}\left\{2 k-1+\frac{2}{\alpha_{n}} \sum_{j=0}^{n} a^{n-j}\left|\beta_{j}\right|\right\} .
$$

\section{Proofs of Theorems}

Proof of Theorem 1. For $P(z)=\sum_{j=0}^{n} a_{j} z^{j}$, we have

$$
P^{\prime}(z)=\sum_{j=0}^{n-1}(j+1) a_{j+1} z^{j}=\sum_{j=0}^{n-1} b_{j} z^{j}=k n a_{n} z^{n-1}+(n-1) a_{n-1} z^{n-2}+\ldots \ldots+2 a_{2} z+a_{1} .
$$

Since

$$
k\left|a_{n}\right| \geq \frac{1}{t}\left|a_{n-1}\right| \geq \frac{1}{t^{2}}\left|a_{n-2}\right| \geq \ldots \ldots . . \geq \frac{1}{t^{n-1}}\left|a_{1}\right| \geq \frac{1}{t^{n}}\left|a_{0}\right|
$$

and

$$
\frac{n-1}{n} \geq \frac{j}{j+1}, j=1,2, \ldots ., n-1,
$$

We have $t \frac{n-1}{n}(j+1)\left|a_{j+1}\right| \geq j\left|a_{j}\right|, j=1,2, \ldots . ., n-2$ i.e $\left|b_{j}\right| \geq \frac{n}{t(n-1)}\left|b_{j-1}\right|, j=1,2, \ldots ., n-2$

and $k\left|b_{n-1}\right| \geq \frac{n}{t(n-1)}\left|b_{n-2}\right|$.

Thus

$$
k\left|b_{n-1}\right| \geq a\left|b_{n-2}\right| \geq a^{2}\left|b_{n-3}\right| \geq \ldots \ldots . . \geq a^{n-1}\left|b_{0}\right|,
$$

where $a=\frac{n}{t(n-1)}$.

Hence by Lemma 2, it follows that all the zeros of the polynomial $P^{\prime}(z)$ of degree (n-1) lie in

$$
|z| \leq \frac{t(n-1)}{n\left|a_{n}\right|}\left\{k\left|a_{n}\right|(\cos \alpha+\sin \alpha+1)-\left|a_{n}\right|+\frac{2 \sin \alpha}{n} \sum_{j=0}^{n-2}\left(\frac{n}{t(n-1)}\right)^{n-1-j}(j+1)\left|a_{j+1}\right|\right\} .
$$

In other words, it follows that all the zeros of $\mathrm{P}(\mathrm{z})$ with modulus greater than

$$
\frac{t(n-1)}{n\left|a_{n}\right|}\left\{k\left|a_{n}\right|(\cos \alpha+\sin \alpha+1)-\left|a_{n}\right|+\frac{2 \sin \alpha}{n} \sum_{j=0}^{n-2}\left(\frac{n}{t(n-1)}\right)^{n-1-j}(j+1)\left|a_{j+1}\right|\right\}
$$

are simple. 
Proof of Theorem 2. For $P(z)=\sum_{j=0}^{n} a_{j} z^{j}$, we have

$$
P^{\prime}(z)=\sum_{j=0}^{n-1}(j+1) a_{j+1} z^{j}=\sum_{j=0}^{n-1} b_{j} z^{j}
$$

Since

$$
k \alpha_{n} \geq \frac{1}{t} \alpha_{n-1} \geq \frac{1}{t^{2}} \alpha_{n-2} \geq \ldots \ldots \geq \frac{1}{t^{n-1}} \alpha_{1} \geq \frac{1}{t^{n}} \alpha_{0} \geq 0,
$$

and

$$
\frac{n-1}{n} \geq \frac{j}{j+1}, j=1,2, \ldots ., n-1,
$$

We have $t \frac{n-1}{n}(j+1) \alpha_{j+1} \geq j \alpha_{j}, j=1,2, \ldots . ., n-2$ i.e $\operatorname{Re} b_{j} \geq \frac{n}{t(n-1)} \operatorname{Re} b_{j-1}, j=1,2, \ldots ., n-2$

and $k \operatorname{Re} b_{n-1} \geq \frac{n}{t(n-1)} \operatorname{Re} b_{n-2}$.

Thus

$$
k \operatorname{Re} b_{n-1} \geq a \operatorname{Re} b_{n-2} \geq \ldots \ldots . a^{n-2} \operatorname{Re} b_{1} \geq a^{n-1} \operatorname{Re} b_{0}
$$

where $a=\frac{n}{t(n-1)}$.

Hence by Lemma 4 , it follows that all the zeros of the polynomial $P^{\prime}(z)$ of degree $(\mathrm{n}-1)$ lie in

$$
|z| \leq \frac{t(n-1)}{n}\left\{2 k-1+\frac{2}{n \alpha_{n}} \sum_{j=0}^{n-1}\left(\frac{n}{t(n-1)}\right)^{n-j}(j+1)\left|\beta_{j+1}\right|\right\}
$$

In other words, it follows that all the zeros of $\mathrm{P}(\mathrm{z})$ with modulus greater than

$$
\frac{t(n-1)}{n}\left\{2 k-1+\frac{2}{n \alpha_{n}} \sum_{j=0}^{n-1}\left(\frac{n}{t(n-1)}\right)^{n-j}(j+1)\left|\beta_{j+1}\right|\right\} \text {. }
$$

are simple.

\section{References}

I. A.Aziz, Q.G.Mohammad, On the zeros of certain class of polynomials and related analytic functions, J.Math.Anal.Appl.,75(1980),495-502.

II. A.Aziz, Q.G.Mohammad, Zero-free regions for polynomials and some generalizations of EnestromKakeya Theorem, Canad. Math.Bull. 27(3),1984,265-272.

III. S.D.Bairagi,Viny Kumar Jain,T.K.Mishra,L.Saha, On the location of the zeros of certain polynomials, Publications De L'Institut Mathematique,Nouvelle serie,tome 99(113) (2016) 287-294.

IV. N.K.Govil, Q.I.Rahman, On the Enestrom-Kakeya theorem,Tohoku Math.J.20(1968),126-136.

V. N.K.Govil, G.N.Mc-Tume, Some extensions of Enestrom-Kakeya Theorem, Int.J.Appl.Math.Vol. 11No.3,2002,245-253.

VI. M.H.Gulzar, Bounds for the zeros and extremal properties of polynomials, Ph.D thesis,University of Kashmir, 2012. 
VII. M. Marden ,Geometry of polynomials, Math.Surveys No.3, Amer.Math.Soc., Providence, Rhode Island, 1966.

VIII. G.V.Milovanovic, D.S.Mitrinovic, T.M.Rassias, Topics in polynomials,Extremal Problems Inequalities,Zeros, World Scientific, Singapore-New Jersy-London-Hongkong, 1994.

IX. Q.I.Rahman, G.Schmeisser,Analytic Theory of Polynomials, Oxford University Press, Oxford ,2002. 